\title{
Data Sampling in Sensor Networks: New Trends in Smart City Applications
}

Andre L. L. Aquino*

Computer Institute, Federal University of Alagoas, Maceio, Alagoas, Brazil

\section{Editorial}

When we think about "Data sampling in sensor networks applied to smart city applications", we depicted several interdisciplinary areas and problems [1]. The central concepts in smart city applications are traditional sensor networks with intelligent devices [2,3]; Internet of things [4]; social sensing [5]; and cloud sensing [6]. Nowadays, the central issue in these scenarios is "how to perform data sampling when these concepts are integrated".

Most smart city applications consider an environment that has a variety of phenomena monitored by sensor networks [2]. In traditional sensor networks, each sensor node can monitor one or more phenomena that are reported, through ad-hoc wireless communication [7], to the sink, these nodes receive and process the data extracting useful information for users [8]. However, in the context of smart city applications, the sensor network becomes a primary element of the IoT concept [9], where we have the several sensors associated with different "things" to monitor the environment and generate data for contextsensitive applications.

Additionally, one of the premises of smart cities is connectivity and iteration among people through the most different technologies. Currently, social networks correspond to one of the leading iteration tools among people, and they are responsible for generating a lot of data. In this context, in a complementary way, applications in smart cities can satisfactorily com-bine data generated by social networks (social sensors) with one produced by sensor networks (physical sensors). These applications are known as social sensors [5]. For instance, we can use a sensor networks monitoring vehicle traffic in a specific area and combine that information with social sensor data watching the people's comments on Twitter about the vehicles traffic in the same area. Thus, the application for traffic management uses both information to improve the user information.

The combination of this information requires an infrastructure for data storage and processing. Thus, the application adopts a cloud solution to favour and assist in this process. In some cases, the applications perform the data storage and query processing directly in the cloud, i.e., the data collected by physical or social sensors are stored in the cloud and available for future queries. This combination of sensor data (physical or social) and cloud computing solutions is known as cloud sensing $[6,10]$. A specific concept in cloud sensing is the fog infrastructure. Fog uses one or more collaborative end-user sensors or near-user edge devices to carry out a substantial amount of storage, rather than stored primarily in cloud data centres.

We observe these characteristics in different applications, for instance, traditional environmental monitoring, vehicular networks [11-14], smart buildings and smart grid [15] applications. So, we identify new trends in data sampling in a smart city as follows:

\section{Internet of things}

i) To design new solutions to integrate different physical sensors or networks through a generic middleware; ii) To design new solutions to allow plug-and-play physical sensors or networks in the smart city application; iii) To design new context-sensitive robust applications allowing the inference and decision oversampled or missed data.

\section{Social sensing}

i) To propose new data sampling algorithms applied to social sensing applications; ii) To propose new data fusion algorithms to infer different behaviours based on social and physical sensor data; and iii) To design new physical sensor applications, where social data sensing improves the inference layer.

\section{Cloud sensing}

i) To design new physical sensor applications, where we integrate the user application queries with data stored in a cloud or fog.

ii) To design new optimization strategies to define when and where to store the data collected by physical sensors; iii) to propose new code offloading strategies to de ne when the cloud of fog have to process a prohibitive task of physical sensors.

Finally, the main issue about data sampling in sensor networks is to integrate different data sampled solutions in a specific application. This integration must be focused on the Internet of Things, Social Sensing, and Could Sensing. By offering solutions for these presented problems, we will improve the smart city applications interoperability.

\section{References}

1. Aquino LL, Junior OS, Frery AC, Albuquerque EL, Mini RAF (2014) muSA Multivariate sampling algorithm for wireless sensor networks. Transactions on Computers 63: 968-978

2. Akyildiz F, Su W, Sankarasubramaniam Y, Cayirci E (2002) A survey on sensor networks, IEEE Communications Magazine 40: 102-114

3. Nakamura EF, Oliveira HABF, Ramos H, Villas LA, Aquino ALL, et al. (2009) A reactive role assignment for data routing in event-based wireless sensor networks. Computer Networks 53: 1980-1996.

4. Bellavista P, Cardone G, Corradi A, Foschini L (2013) Convergence of MANET and WSN in IoT urban scenarios. IEEE Sensors Journal 13: 3558-3567.

5. Wang D, Abdelzaher T, Kaplan L (2015) Social Sensing: Building Reliable Systems on Unreliable Data. Morgan Kaufmann ebook, P: 232.

6. Han Q, Liang S, Zhang H (2015) Mobile cloud sensing, big data, and $5 \mathrm{~g}$ networks make an intelligent and smart world, IEEE Network 29: 40-45.

*Corresponding author: Andre L. L. Aquino, Computer Institute, Federa University of Alagoas, Maceio, Alagoas, Brazil, Tel: +55 82 32141401; E-mail: alla@laccan.ufal.br

Received April 02, 2018; Accepted April 03, 2018; Published April 13, 2018

Citation: Aquino ALL (2018) Data Sampling in Sensor Networks: New Trends in Smart City Applications. Int J Sens Netw Data Commun 7: e115. doi: 10.4172/20904886.1000e115

Copyright: @ 2018 Aquino ALL. This is an open-access article distributed under the terms of the Creative Commons Attribution License, which permits unrestricted use, distribution, and reproduction in any medium, provided the original author and source are credited. 
Citation: Aquino ALL (2018) Data Sampling in Sensor Networks: New Trends in Smart City Applications. Int J Sens Netw Data Commun 7: e115. doi: 10.4172/2090-4886.1000e115

Page 2 of 2

7. Cabral RS, Aquino ALL, Frery AC, Rosso OA, Ramrez JA (2013) Structural changes in data communication in wireless sensor networks. Central European J Physics 11: 1645-1652.

8. Aquino LL, Nakamura EF (2009) Data centric sensor stream reduction for realtime applications in wireless sensor networks. Sensors Basel 9: 9666-9688.

9. Lima DHS, Aquino ALL, Ramos HS, Almeida ES, Rodrigues JJPC (2014) Oasys: an opportunistic and agile system to detect free on-street parking using intelligent boards embedded in surveillance 3 cameras. J Network and Computer Applications 46: 241-249.

10. wahab AS, Hamdaoui B, Guizani M, Rayes A (2014) Enabling smart cloud services through remote sensing: An internet of everything en-abler. IEEE Internet of Things Journal 1: 276-288.

11. Maia G, Villas LA, Aquino ALL, Viana AC, Loureiro AAF (2015) A rate control video dissemination solution for extremely dynamic vehicular ad hoc networks. Performance Evaluation 87: 3-18

12. Silva CM, Aquino ALL, Meira-Jr W (2015) Smart traffic light for low traffic conditions, Mobile Networks and Applications. Sensors (basel) 20: 285.

13. Silva CM, Aquino ALL, Meira-Jr W (2015) Deployment of roadside units based on partial mobility information. Computer Communications 60: 28-39.

14. Aquino ALL, Cavalcante TSG, Almeida ES, Frery AC, OA Rosso (2015) Characterization of vehicle behavior with information theory, The European Physical J B. Condensed Matter Physics 88: 257.

15. Aquino ALL, Ramos HS, Frery AC, Viana LP, Cav-alcante TSG, et al. (2016) Characterization of electric load with information theory quanti ers, Physica $A$ 465: $277-284$ 